\title{
Enhanced hole transport in InGaN/GaN multiple quantum well light-emitting diodes with a p-type doped quantum barrier
}

\author{
Yun Ji, ${ }^{1}$ Zi-Hui Zhang, ${ }^{1}$ Swee Tiam Tan, ${ }^{1}$ Zhen Gang Ju, ${ }^{1}$ Zabu Kyaw, ${ }^{1}$ Namig Hasanov, ${ }^{1}$ \\ Wei Liu, ${ }^{1}$ Xiao Wei Sun, ${ }^{1,2,5}$ and Hilmi Volkan Demir ${ }^{1,3,4,6}$ \\ ${ }^{1}$ Luminous! Centre of Excellence for Semiconductor Lighting and Displays, School of Electrical and Electronic Engineering, \\ Nanyang Technological University, 50 Nanyang Avenue, Singapore 639798, Singapore \\ ${ }^{2}$ Department of Applied Physics, College of Science, and Tianjin Key Laboratory of Low-Dimensional Functional Material \\ Physics and Fabrication Technology, Tianjin University, Tianjin 300072, China \\ ${ }^{3}$ School of Physics and Mathematical Sciences, Nanyang Technological University, 21 Nanyang Link, Singapore 637371, Singapore \\ ${ }^{4}$ Department of Electrical and Electronics, Department of Physics, and National \\ Nanotechnology Research Center-Institute of Material Science \\ and Nanotechnology, Bilkent University, Ankara TR-06800, Turkey \\ ${ }^{5}$ e-mail: exwsun@ntu.edu.sg \\ ${ }^{6}$ e-mail: hvdemir@ntu.edu.sg
}

Received November 6, 2012; accepted November 29, 2012; posted December 10, 2012 (Doc. ID 179363); published January 11, 2013

\begin{abstract}
We study hole transport behavior of InGaN/GaN light-emitting diodes with the dual wavelength emission method. It is found that at low injection levels, light emission is mainly from quantum wells near p-GaN, indicating that hole transport depth is limited in the active region. Emission from deeper wells only occurs under high current injection. However, with Mg-doped quantum barriers, holes penetrate deeper within the active region even under low injection, increasing the radiative recombination. Moreover, the improved hole transport leads to reduced forward voltage and enhanced light generation. This is also verified by numerical analysis of hole distribution and energy band structure. (c) 2013 Optical Society of America

OCIS codes: $230.3670,230.5590,160.6000$.
\end{abstract}

Nitride-based high-power light-emitting diodes (LEDs) are considered the new generation lighting sources due to their advantages in power consumption, energy efficiency, lifetime, and color quality [1,2]. To date tremendous efforts have been devoted to the development of high-brightness GaN-based LEDs [3-5]. Lateral hole spreading is one of the challenges that limit the performance of LED chips. We have illustrated the enhanced hole spreading performance by applying a $\mathrm{p} / \mathrm{n} / \mathrm{p} / \mathrm{n} / \mathrm{p}$-GaN multilayer structure in the p-region [] ]. Another important issue to be addressed is transporting the hole vertically into the depths of multiple quantum wells (MQWs). Due to the difficulty of hole transporting [7], excessive electron injection is commonly adopted to maximize radiative recombination, resulting in electron overflow. Hence, an electron blocking layer is necessary to prevent electrons from leaking into the $\mathrm{p}$-GaN region. Different approaches to enhancing hole transportation have been reported, including replacing GaN barriers with AlGaN quantum barriers (QBs) []ㅛ, varying quantum well (QW) thickness [9], graded-composition QBs [10], and p-type doped last QBBs [11]. Also, Liu et al. [12] and Han et al. [13] examined the effect of a p-type doped barrier on hole transport and hole distribution. However, hole transport behavior under different current injection levels has not been studied or reported to date.

In this work, we investigated the hole transporting behavior in the MQW region with a dual-wavelength emission study under different current injection levels. The MQWs were grown at different temperatures intentionally to incorporate different indium compositions, resulting in dual-wavelength emission. The penetration depth of holes is reflected through emission intensity at different wavelengths. In this scheme, it was found that holes could only penetrate into shallow depths in the MQWs near the p-GaN region. By doping the last three QBs with Mg, the light emission from deeper MQWs was enhanced, indicating that the recombination region shifts toward the n-GaN layer.

The LED epiwafers were grown on (0001) $c$-plane sapphire substrates using metal-organic chemical vapor deposition. Sample I consists of a $30 \mathrm{~nm}$ thick lowtemperature GaN nucleation layer, a $150 \mathrm{~nm}$ thick interlayer [14], a $4 \mu \mathrm{m}$ thick unintentionally doped GaN layer, a $2 \mu \mathrm{m}$ thick Si-doped n-GaN layer (doping concentration $5 \times 10^{18} \mathrm{~cm}^{-3}$ ), eight pairs of InGaN/GaN MQWs, and a $150 \mathrm{~nm}$ thick Mg-doped $\mathrm{p}$-GaN layer (doping concentration $\left(3 \times 10^{17} \mathrm{~cm}^{-3}\right)$, as shown in Fig. 1. The first five QWs close to n-GaN (referred to as "deeper QWs") were gown

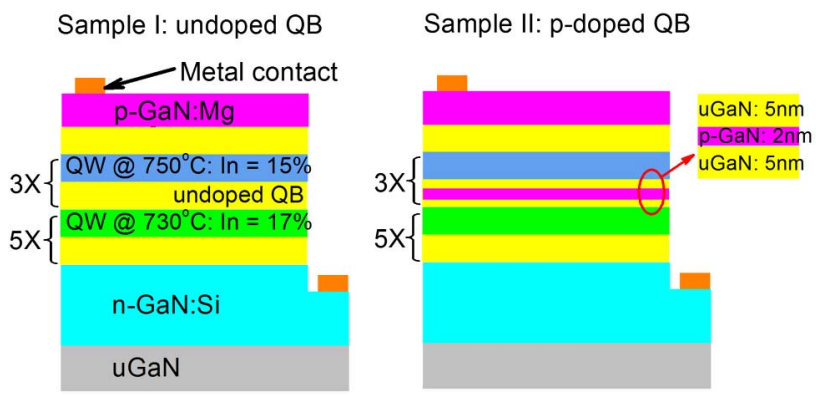

Fig. 1. (Color online) Schematic diagram of the LED structures: Sample I with undoped QBs and Sample II with p-doped QBs. 


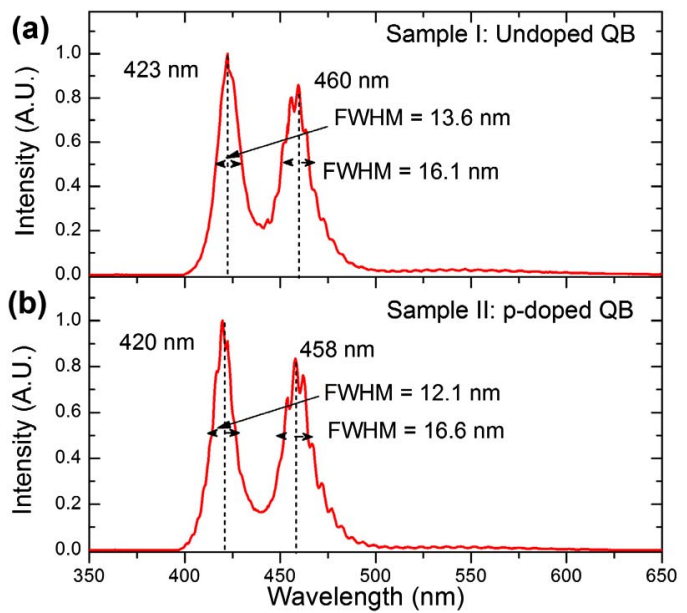

Fig. 2. (Color online) PL spectra of (a) Sample I with undoped QBs and (b) Sample II with the last three barriers doped with Mg.

at $730^{\circ} \mathrm{C}$, followed by three QWs grown at $750^{\circ} \mathrm{C}$. Sample II has the same epilayer structure, except that a $2 \mathrm{~nm}$ p-doped GaN layer with the same doping concentration as the p-GaN layer was inserted into the last three QBs. The samples were further fabricated into device chips using a standard device fabrication process, with a device area of $350 \mu \mathrm{m} \times 350 \mu \mathrm{m}$.

The photoluminescence (PL) spectra in Figs. 2(a) and 2(b) show that both samples have two distinguished emission peaks due to different $\mathrm{QW}$ growth temperatures. The shorter wavelength peaks, centered at $423 \mathrm{~nm}$ for Sample I and $420 \mathrm{~nm}$ for Sample II, originate from the last three QWs, which have an indium content of $15 \%$. The QWs grown at $730^{\circ} \mathrm{C}$ possess a higher indium content of about $17 \%$, which results in peak emissions at 460 and $458 \mathrm{~nm}$ for Samples I and II, respectively. Judging from the narrow full width at half-maximum (FWHM) of the emission peaks, which is between 12

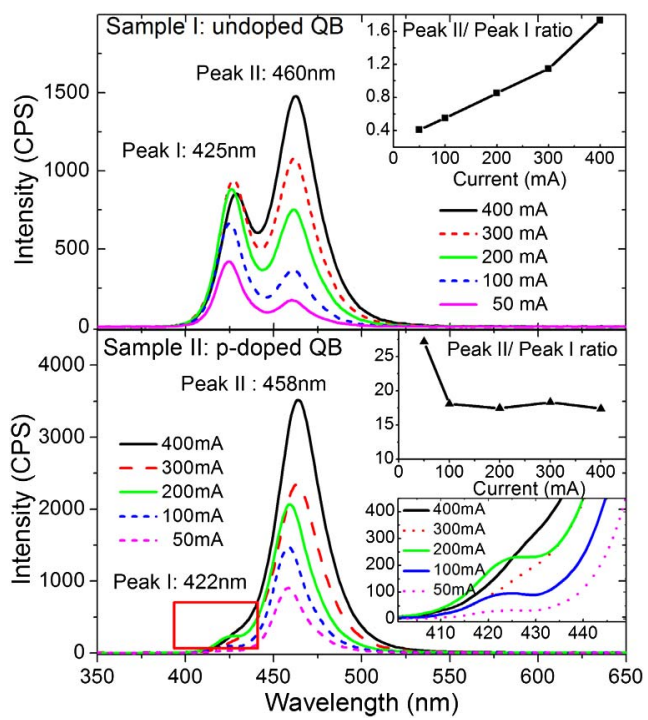

Fig. 3. (Color online) Electroluminescence spectra of LED samples driven at different current levels. Top: Sample I without p-doped QBs (inset: ratio of peak intensity at 460 and $425 \mathrm{~nm}$ ). Bottom: Sample II with p-doped QBs (top inset: ratio of peak intensity at 458 and $422 \mathrm{~nm}$; bottom inset: zoom-in view of EL peak at $422 \mathrm{~nm}$ ). and $16 \mathrm{~nm}$, the MQW structure was not degraded by the p-doping in the $\mathrm{QB}$.

The EL spectra were captured at different injection currents, as shown in Fig. 3. For Sample I, at a lower injection current level, the emission is dominated at $425 \mathrm{~nm}$, together with a weak emission peak at $460 \mathrm{~nm}$. This suggests that the radiative recombination of electrons and holes mainly occurs at the QWs near p-GaN. Only a small portion of holes were then able to travel into the deep $\mathrm{QW}$ region and recombine with electrons, even though the electron concentration is higher in these QWs.

With an injection current above $300 \mathrm{~mA}$, the emission at $425 \mathrm{~nm}$ is saturated, whereas the emission peak at $460 \mathrm{~nm}$ is substantially enhanced. This means that at high injection levels, the hole concentration in QWs near $\mathrm{p}-\mathrm{GaN}$ is close to the saturation limit. With a further increased bias, the radiative recombination of electrons and holes is saturated. The excess holes tend to overflow into deeper QWs, leading to the rapid increase of light emission at $460 \mathrm{~nm}$. For the p-type doped QB sample, the emission at the short wavelength peak, from the last three pairs of QWs, was minor compared with the long wavelength emission from the deeper QWs, as the p-type doping in the last three QBs enhanced the hole conductivity and made it easier for holes to penetrate further into the MQW region, even at low injection levels.
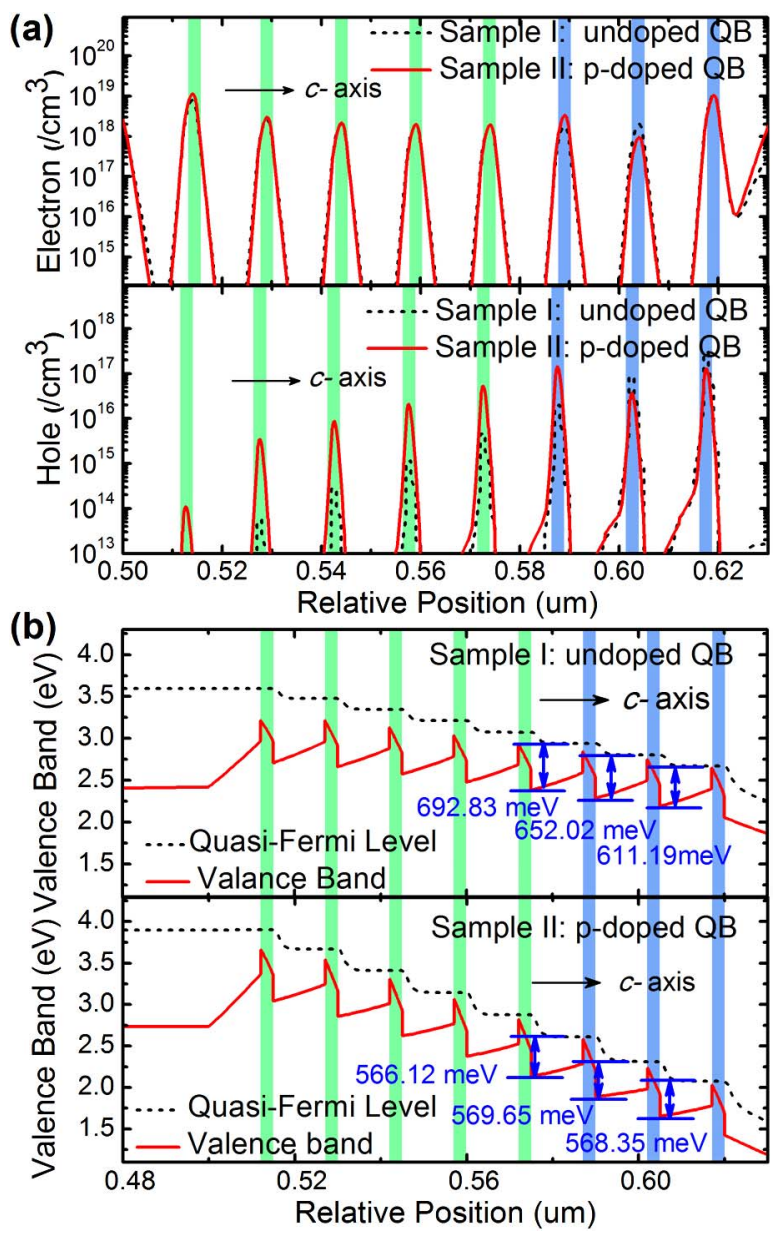

Fig. 4. (Color online) (a) Simulated electron and hole distributions within MQW regions and (b) energy band diagrams for LED structures with and without p-type doped QBs. 

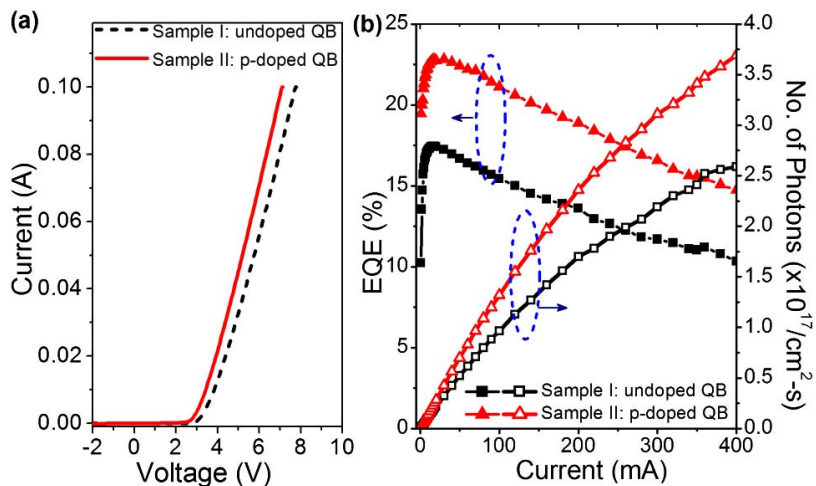

Fig. 5. (Color online) (a) $I-V$ characteristics and (b) EQE and number of photons measured for Sample I with undoped QBs and Sample II with p-type doped QBs.

To further verify this conclusion, the device structures were numerically simulated using APSYS software. The structures studied were the same as the epitaxial LED design. The indium compositions in the first five QWs and the last three QWs were set to $15 \%$ and $17 \%$, corresponding to the peak emission wavelengths at 425 and $460 \mathrm{~nm}$, respectively. The Auger recombination coefficient is taken to be $1 \times 10^{42} \mathrm{~m}^{6} / \mathrm{s}$, and $40 \%$ of the polarization charge was assumed for crystal relaxation. Other parameters used in the simulation can be found in the literature [15].

The calculated carrier concentration at $120 \mathrm{~A} / \mathrm{m}$ (corresponding to the high injection level) shows that the structure with the last three barriers doped with $\mathrm{Mg}$ has a more uniform hole distribution, as illustrated in Fig. 4(a). For Sample I with undoped barriers, the hole concentration reduces substantially as holes travel deeper into the QWs. After doping the last three barriers with $\mathrm{Mg}$, the hole concentrations in the deeper QWs are at least one order of magnitude higher, resulting in more chances for electron-hole recombination in these QWs. Meanwhile, there is a small amount of decrease in the hole concentration in the last two QWs. In view of the relatively high hole concentration in these QWs, this small reduction is helpful in avoiding hole crowding. By analyzing the energy band structure in Fig. 4(b), it is found that the energy barriers between the quasi-Fermi level and the valance band for the doped barrier are lower (568.35, 569.6 and $566.12 \mathrm{meV}$, respectively) compared to those of undoped QBs (611.19, 652.02, and $692.83 \mathrm{meV}$, respectively), as the p-type doping shifts the quasi-Fermi level down toward the valance band, bringing down the potential barrier for holes. Hence, the hole conductivity is improved.

The enhanced hole conductivity is also evidenced by the improved $I-V$ characteristics, as shown in Fig. 5(a). The forward voltage is reduced for Sample II, due to a better hole conductivity with the Mg-doped QBs. Also, the optical power output and the external quantum efficiency (EQE) are enhanced, as illustrated in Fig. 5(b). The maximum EQE is increased by $30.9 \%$ with p-doped QBs, while the efficiency droop measured at $350 \mathrm{~mA}$ is reduced from $36.71 \%$ to $31.62 \%$. The improved performance of EQE and efficiency droop is attributed to the better hole transportation.

In conclusion, the hole transporting behavior of a partially p-doped QB LED structure was studied through the double peak emission method. Hole conductivity was enhanced by partially doping the QBs into p-type, leading to a more uniform distribution of holes. Meanwhile, the hole crowding in QWs close to p-GaN is reduced. The enhanced hole conductivity is attributed to the reduced energy barrier due to the $\mathrm{Mg}$ doping. The additional p-type doping results in improved electrical performance and enhanced light output from the LED device. Such p-type doping of QBs hold great promise for LED performance enhancement.

We gratefully acknowledge that this work is supported by the Singapore National Research Foundation under Grant Nos. NRF-RF-2009-09 and NRF-CRP-6-2010-2 and the Singapore Agency for Science, Technology and Research (A*STAR) SERC under Grant No. 112120 2009. The work is also supported by the National Natural Science Foundation of China (NSFC) (Project Nos. 61006037, 61177014, and 61076015) and Tianjin Natural Science Foundation (Project Nos. 11JCZDJC 21900 and 11JCYDJC25800).

\section{References}

1. S. Pimputkar, J. S. Speck, S. P. DenBaars, and S. Nakamura, Nat. Photonics 3, 180 (2009).

2. S. T. Tan, X. W. Sun, H. V. Demir, and S. P. DenBaars, IEEE Photonics J. 4, 613 (2012).

3. X. Fu, B. Zhang, X. Kang, J. Deng, C. Xiong, T. Dai, X. Jiang, T. Yu, Z. Chen, and G. Y. Zhang, Opt. Express 19, A1104 (2011).

4. H. K. Cho, J. Jang, J.-H. Choi, J. Choi, J. Kim, J. S. Lee, B. Lee, Y. H. Choe, K.-D. Lee, S. H. Kim, K. Lee, S.-K. Kim, and Y.-H. Lee, Opt. Express 14, 8654 (2006).

5. C.-Y. Huang, H.-M. Ku, C.-Z. Liao, and S. Chao, Opt. Express 18, 10674 (2010).

6. Z. H. Zhang, S. T. Tan, W. Liu, Z. Ju, K. Zheng, Z. Kyaw, Y. Ji, N. Hasanov, X. W. Sun, and H. V. Demir are preparing a paper to be called, "Improved InGaN/GaN light-emitting diodes with a p-GaN/n-GaN/p-GaN/n-GaN/p-GaN currentspreading layer,"

7. R. Charash, P. P. Maaskant, L. Lewis, C. McAleese, M. J. Kappers, C. J. Humphreys, and B. Corbett, Appl. Phys. Lett. 95, 151103 (2009).

8. J.-Y. Chang, M.-C. Tsai, and Y.-K. Kuo, Opt. Lett. 35, 1368 (2010).

9. Y. L. Li, Y. R. Huang, and Y. H. Lai, Appl. Phys. Lett. 91, 181113 (2007).

10. C. H. Wang, S. P. Chang, P. H. Ku, J. C. Li, Y. P. Lan, C. C. Lin, H. C. Yang, H. C. Kuo, T. C. Lu, S. C. Wang, and C. Y. Chang, Appl. Phys. Lett. 99, 171106 (2011).

11. Y.-K. Kuo, M.-C. Tsai, S.-H. Yen, T.-C. Hsu, and Y.-J. Shen, IEEE J. Quantum Electron. 46, 1214 (2010).

12. J. P. Liu, J. H. Ryou, R. D. Dupuis, J. Han, G. D. Shen, and H. B. Wang, Appl. Phys. Lett. 93, 021102 (2008).

13. S.-H. Han, C.-Y. Cho, S.-J. Lee, T.-Y. Park, T.-H. Kim, S. H. Park, S. W. Kang, J. W. Kim, Y. C. Kim, and S.-J. Park, Appl. Phys. Lett. 96, 051113 (2010).

14. Z. G. Ju, S. T. Tan, Z. H. Zhang, Y. Ji, Z. Kyaw, Y. Dikme, X. W. Sun, and H. V. Demir, Appl. Phys. Lett. 100, 123503 (2012).

15. I. Vurgaftman and J. R. Meyer, J. Appl. Phys. 94, 3675 (2003). 